\title{
What kinds of coordinate can keep the Hawking temperature invariant for the static spherically symmetric black hole?
}

\author{
Chikun Ding and Jiliang Jing* \\ Institute of Physics and Department of Physics, \\ Hunan Normal University, Changsha, \\ Hunan 410081, P. R. China \\ and \\ Key Laboratory of Low Dimensional Quantum Structures \\ and Quantum Control of Ministry of Education, \\ Hunan Normal University , Changsha, \\ Hunan 410081, P.R. China
}

\begin{abstract}
By studying the Hawking radiation of the most general static spherically symmetric black hole arising from scalar and Dirac particles tunnelling, we find the Hawking temperature is invariant in the general coordinate representation (11), which satisfies two conditions: a) its radial coordinate transformation is regular at the event horizon; and b) there is a time-like Killing vector.
\end{abstract}

PACS numbers: 04.70.Dy, 04.62.+v

\section{INTRODUCTION}

In recent years, a semi-classical method of modeling Hawking radiation as a tunnelling effect has been developed and has excited a lot of interest [1, 2, 3, 4, 15, 6, 7, 8, 9, 10, 11, 12, 13, 14, 15, 16, 17, 18, 19, 20, 21,22 ]. Tunnelling provides not only a useful verification of thermodynamic properties of black holes but also an alternate conceptual means for understanding the underlying physical process of black hole radiation. In the tunnelling approach, the particles are allowed to follow classically forbidden trajectories, by starting just behind the horizon onward to infinity. The particles must then travel necessarily back in time, since the horizon is locally to the future of the static or stationary external region. The classical, one-particle action becomes complex, signaling the classical impossibility of the motion, and gives the amplitude an imaginary part, provides a semi-classical approximation to free field propagators. In general the tunnelling methods involve calculating the imaginary part of the action $I$ for the (classically forbidden) process of s-wave emission across the horizon, which in turn is related to the Boltzmann factor for emission at the Hawking temperature, i.e.

$$
\Gamma \propto e^{-2 \operatorname{Im} I}=e^{-E / T_{H}},
$$

where $T_{H}$ is the Hawking temperature of the black hole, $E$ is the energy of the tunnelling particles.

\footnotetext{
${ }^{*}$ Corresponding author, Electronic address: jljing@hunnu.edu.cn
} 
There are two different approaches that are used to calculate the imaginary part of the action for the emitted particle. The first method developed was the Null Geodesic Method used by Parikh and Wilczek [3]; The other approach is the Hamilton-Jacobi Ansatz used by Agheben et al [4] which is an extension of the complex path analysis of Padmanabhan et al [5, 6, 7]. For the Hamilton-Jacobi ansatz it is assumed that the action of the emitted scalar particle satisfies the relativistic Hamilton-Jacobi equation. From the symmetries of the metric one picks an appropriate ansatz for the form of the action and plugs it into the relativistic Hamilton-Jacobi equation to solve.

Since a black hole has a well defined temperature it should radiate all types of particles like a black body at that temperature. The emission spectrum therefore contains particles of all spins such as Dirac particles. In this paper, we will use the Hamilton-Jacobi ansatz method to calculate the Hawking temperature.

Can the Hawking temperature keep invariant under any coordinate transformation? At the first glance, the Hawking temperature is invariant. However, this invariance has been lost in the following isotropic coordinate [4, 8] for the Schwarzschild black hole

$$
t \rightarrow t, \quad r \rightarrow \rho, \quad \ln \rho=\int \frac{d r}{r \sqrt{1-\frac{2 M}{r}}} .
$$

And so the line element of the Schwarzschild black hole becomes

$$
d s^{2}=-\left(\frac{2 \rho-M}{2 \rho+M}\right)^{2} d t^{2}+\left(\frac{2 \rho+M}{2 \rho}\right)^{4} d \rho^{2}+\frac{(2 \rho+M)^{4}}{16 \rho^{2}} d \Omega^{2}
$$

and the horizon $\rho_{H}=M / 2$. Substituting it and $\phi=e^{i[-E t+W(\rho)+J(\theta, \varphi)] / \hbar}$ into Klein-Gordon equation

$$
\frac{1}{\sqrt{-g}} \partial_{\mu}\left(\sqrt{-g} g^{\mu \nu} \partial \nu \phi\right)-\frac{m^{2}}{\hbar^{2}} \phi=0
$$

we can obtain

$$
\begin{aligned}
\operatorname{Im} W_{ \pm}(\rho) & = \pm \operatorname{Im}\left[\int \frac{(2 \rho+M)^{3} d \rho}{4 \rho^{2}(2 \rho-M)} \sqrt{E^{2}-\left(\frac{2 \rho-M}{2 \rho+M}\right)^{2}\left(m^{2}+g^{i j} J_{i} J_{j}\right)}\right] \\
& = \pm 4 \pi M E
\end{aligned}
$$

The probability is [5, 6, 7$]$

$$
\Gamma=\frac{\Gamma_{\text {out }}}{\Gamma_{\text {in }}} \propto \exp \left[-4 \operatorname{Im} W_{+}\right]=\exp [-16 \pi M E]=\exp \left[-\frac{E}{T_{H}}\right],
$$

since $W_{-}=-W_{+}$. Then the black hole's temperature is [8]

$$
T_{H}=\frac{1}{16 \pi M}
$$

which is one-half of the standard Hawking temperature $T_{H}=1 / 8 \pi M$. The example tell us that the invariance is missing in the isotropic coordinate! The reason for the phenomenon comes from the coordinate transformation (2) itself. In the radial coordinate transformation

$$
\ln \rho=\int \frac{d r}{r \sqrt{1-\frac{2 M}{r}}}=\int F(r) d r
$$


the function $F(r)=\frac{1}{r \sqrt{1-2 M / r}}$ has singularity at the horizon $r=2 M$. So it needs to discuss that in which coordinates can Hawking temperature be invariant.

The purpose of this manuscript is to investigate the invariance of the Hawking temperature of the most general static spherically symmetric black hole from scalar and Dirac particles tunnelling in a general coordinate representations. In order to do that, we introduce the metrics of the static spherically symmetric black in the two coordinates: Schwarzschild-like and a general coordinates. This general coordinate should satisfy two conditions: a) its radial coordinate transformation is regular at the event horizon; b) there exists a time-like Killing vector.

The paper is organized as follows. In Sec. 2 the different coordinate representations for the general static spherically symmetric black hole are presented. In Sec. 3 the Hawking temperature of the general static spherically symmetric black hole for scalar particles tunnelling is investigated. In Sec. 4 the Hawking temperature of the general static spherically symmetric black hole from Dirac particles tunnelling is studied. The last section is devoted to a summary.

\section{COORDINATE REPRESENTATIONS FOR GENERAL STATIC SPHERICALLY SYMMETRIC BLACK HOLE}

In this section we introduce two kinds of the coordinate representations for the general static spherically symmetric black hole, i. e. the Schwarzschild-like and a general coordinates.

\section{A. Schwarzschild-like coordinate representation}

In Schwarzschild-like coordinate the line element for the most general static spherically symmetric black hole in four dimensional spacetime is described by

$$
d s^{2}=-f(r) d t_{s}^{2}+\frac{1}{g(r)} d r^{2}+R(r)\left(d \theta^{2}+\sin ^{2} \theta d \varphi^{2}\right),
$$

where $f(r), g(r)$ and $R(r)$ are functions of $r$, and $t_{s}$ is the Schwarzschild-like time coordinate.

Because the spacetime (9) is a static and spherically symmetric one, a time-like Killing vector field $\xi^{\mu}=$ $(1,0,0,0)$ exists. An interesting feature of the black hole worthy of note is that the norm of the Killing field $\xi^{\mu}$ is zero on the event horizon $r_{H}$ since the horizon is a null surface and the vector $\xi^{\mu}$ is normal to the horizon. Then, for the non-extreme case we have $f(r)=f_{1}(r)\left(r-r_{H}\right)$ and $g(r)=g_{1}(r)\left(r-r_{H}\right)$, where $f_{1}(r)$ and $g_{1}(r)$ are regular functions in the region $r_{H}<r<\infty$ and their values are nonzero on the outermost event horizon.

\section{B. General coordinate representation}

In order to insure that there is a time-like Killing vector in the spacetime, the most general coordinate $(v, u, \theta, \varphi)$ that transform from the Schwarzschild-like coordinate (9) is

$$
v=\lambda t_{s}+\int d r G(r), \quad u=\int d r F(r),
$$


where $v$ is the time coordinate, $u$ is the radial one, and the angular coordinates remain unchanged; $\lambda$ is an arbitrary nonzero constant which re-scales the time; $G$ is arbitrary functions of $r$ and $F$ is a regular function of $r$. The line element (9) in the new coordinate becomes

$$
\begin{aligned}
d s^{2}= & -\frac{f(r(u))}{\lambda^{2}} d v^{2}+2 \frac{f(r(u)) G(r(u))}{\lambda^{2} F(r(u))} d u d v \\
& +\frac{\lambda^{2}-f(r(u)) g(r(u)) G^{2}(r(u))}{\lambda^{2} g(r(u)) F^{2}(r(u))} d u^{2}+R(r(u))\left(d \theta^{2}+\sin ^{2} \theta d \varphi^{2}\right) .
\end{aligned}
$$

We now show that two well-known coordinates, the Painlevé and Lemaitre coordinates, are the spacial cases of the metric (11).

\section{Painlevé coordinate representation}

In the transformation (10), one sets $\lambda=1, G(r)=\sqrt{\frac{1-g(r)}{f(r) g(r)}}$ and $F(r)=1$, the line element (11) becomes the Painlevé coordinate representation [2, 23]

$$
d s^{2}=-f(r) d t^{2}+2 \sqrt{\frac{f(r)(1-g(r))}{g(r)}} d t d r+d r^{2}+R(r)\left(d \theta^{2}+\sin ^{2} \theta d \varphi^{2}\right),
$$

where $t$ is the Panlevé time. The metric (12) has no singularity at $\mathrm{g}(\mathrm{r})=0$, so the metric is regular at the horizon of the black hole. That is to say, the coordinate complies with perspective of a free-falling observer, who is expected to experience nothing out of the ordinary upon passing through the event horizon.

\section{Lemaitre coordinate representation}

In the transformation (10), one sets $\lambda=1, G(r)=\frac{1}{2} \sqrt{\frac{g(r)}{f(r)(1-g(r))}}+\sqrt{\frac{1-g(r)}{f(r) g(r)}}$ and $F(r)=\frac{1}{2} \sqrt{\frac{g(r)}{f(r)(1-g(r))}}$, the line element (11) at present becomes the Lemaitre coordinate representation [23, 24]

$$
d s^{2}=-f(r)\left[d V^{2}+d U^{2}\right]+2 \frac{f(r)(2-g(r))}{g(r)} d V d U+R(r)\left(d \theta^{2}+\sin ^{2} \theta d \varphi^{2}\right),
$$

where $U$ is Lemaitre radial coordinate and $V$ is the Lemaitre time one. We can see that the Lemaitre coordinate is time-dependant system, suggests that there could be a genuine particle production.

\section{TEMPERATURE OF GENERAL STATIC SPHERICALLY SYMMETRIC BLACK HOLE FROM SCALAR PARTICLES TUNNELLING}

We now investigate scalar particles tunnelling of general static spherically symmetric black hole.

\section{A. Scalar particles tunnelling in Schwarzschild-like coordinate}

Applying the WKB approximation

$$
\phi(t, r, \theta, \varphi)=\exp \left[\frac{i}{\hbar} I(t, r, \theta, \varphi)+I_{1}(t, r, \theta, \varphi)+\mathcal{O}(\hbar)\right],
$$


to the Klein-Gordon equation (4), then, to leading order in $\hbar$ we get the following relativistic Hamilton-Jacobi equation

$$
g^{\mu \nu}\left(\partial_{\mu} I \partial_{\nu} I\right)+m^{2}=0
$$

As usual, due to the symmetries of the metric (9) and neglecting the effects of the self-gravitation of the particles, there exists a solution in the form

$$
I=-E t_{s}+W(r)+J(\theta, \varphi)
$$

Inserting Eq. (16) and the metric (9) into the Hamilton-Jacobi equation (15), we find

$$
W_{ \pm}(r)= \pm \int \frac{d r}{\sqrt{f(r) g(r)}} \sqrt{E^{2}-f(r)\left(m^{2}+g^{i j} J_{i} J_{j}\right)}
$$

where $J_{i}=\partial_{i} I, i=\theta, \varphi$. One solution of the Eq. (17) corresponds to the scalar particles moving away from the black hole (i.e. "+" outgoing) and the other solution corresponds to particles moving toward the black hole (i.e. "-" incoming). Imaginary parts of the action can only come due the pole at the horizon. The probability of a particle tunnelling from inside to outside the horizon is [5, 6, 6$]$

$$
\Gamma=\frac{\Gamma_{\text {out }}}{\Gamma_{\text {in }}} \propto \exp \left[-4 \operatorname{Im} W_{+}\right]=\exp \left[-\frac{E}{T_{H}}\right],
$$

since $W_{-}=-W_{+}$. Integrating around the pole at the horizon leads to

$$
\operatorname{Im} W_{+}=\frac{\pi E}{\sqrt{f^{\prime}\left(r_{H}\right) g^{\prime}\left(r_{H}\right)}} .
$$

Substituting (19) into (18), we obtain the Hawking temperature

$$
T_{H}=\frac{\sqrt{f^{\prime}\left(r_{H}\right) g^{\prime}\left(r_{H}\right)}}{4 \pi},
$$

which shows that the temperature of general static spherically symmetric black hole is the same as previous works [1, 2].

\section{B. Scalar particles tunnelling in general coordinate}

Here we study the scalar tunnelling in a general coordinate (11). Employing the ansatz

$$
I=-E v+W(u)+J(\theta, \varphi)
$$

and substituting the metric (11) into the Hamilton-Jacobi equation (15), we obtain

$$
\begin{aligned}
& {\left[g(r(u)) G^{2}(r(u))-\frac{\lambda^{2}}{f(r(u))}\right] E^{2}-2 g(r(u)) G(r(u)) F(r(u)) E W^{\prime}(u)} \\
& +g(r(u)) F^{2}(r(u))\left[W^{\prime}(u)\right]^{2}+g^{i j} J_{i} J_{j}+m^{2}=0 .
\end{aligned}
$$

Then $W^{\prime}(u)$ is

$$
W_{ \pm}^{\prime}(u)=\frac{G(r(u))}{F(r(u))} E \pm \frac{\sqrt{\lambda^{2} E^{2}-f(r(u))\left[g^{i j} J_{i} J_{j}+m^{2}\right]}}{F(r(u)) \sqrt{f(r(u)) g(r(u))}}
$$

We will study the temperature for two cases: $G(r(u))$ is regular function and $G(r(u))$ has a pole at horizon. 


\section{1. $G(r(u))$ is regular function at horizon}

When $G(r(u))$ is regular at horizon, $g_{u u}$ of metric (11) shows that there is still a coordinate singularity at the horizon $r_{H}$. From equation (23) we get

$$
\begin{aligned}
\operatorname{Im} W_{ \pm}(u) & =\operatorname{Im} \int d u\left\{\frac{G(r(u))}{F(r(u))} E \pm \frac{\sqrt{\lambda^{2} E^{2}-f(r(u))\left[g^{i j} J_{i} J_{j}+m^{2}\right]}}{F(r(u)) \sqrt{f(r(u)) g(r(u))}}\right\} \\
& = \pm \frac{\lambda E \pi}{\sqrt{f^{\prime}\left(r_{H}\right) g^{\prime}\left(r_{H}\right)}} .
\end{aligned}
$$

We can see the $\operatorname{Im} W_{ \pm}(u)$ are like those in Schwarzschild-like coordinate. Using

$$
\Gamma \propto \exp \left[-4 \operatorname{Im} W_{+}\right]=\exp \left[-\frac{\lambda E}{T_{H}}\right]
$$

we can recover Hawking temperature (20).

\section{2. $G(r(u))$ has a pole at horizon}

When $G(r(u))$ has a pole at horizon, without loss of generality, it can be expressed as $G(r(u))=$

$\frac{C(r(u))}{\sqrt{f(r(u)) g(r(u))}}+D(r(u))$, where $C(r(u))$ and $D(r(u))$ are the regular functions at horizon. From Eq. (23), we obtain

$$
\begin{aligned}
\operatorname{Im} W_{ \pm}(u) & =\operatorname{Im} \int d u\left\{\frac{D(r(u))}{F(r(u))} E+\frac{C(r(u)) E \pm \sqrt{\lambda^{2} E^{2}-f(r(u))\left[g^{i j} J_{i} J_{j}+m^{2}\right]}}{F(r(u)) \sqrt{f(r(u)) g(r(u))}}\right\} \\
& =\frac{\left(\frac{C\left(r_{H}\right)}{\lambda} \pm 1\right) \lambda E \pi}{\sqrt{f^{\prime}\left(r_{H}\right) g^{\prime}\left(r_{H}\right)}} .
\end{aligned}
$$

In the following, we will consider two cases, i. e., $C\left(r_{H}\right) \neq \lambda$ and $C\left(r_{H}\right)=\lambda$ :

i) If $C\left(r_{H}\right) \neq \lambda$, after substituting $G(r(u))=\frac{C(r(u))}{\sqrt{f(r(u)) g(r(u))}}+D(r(u))$ into $g_{u u}$ of metric (11), it is easy to see that there is still a coordinate singularity at horizon $r_{H}$, and the probabilities are

$$
\Gamma_{\text {out }} \propto \exp \left[-2 \frac{\left(\frac{C\left(r_{H}\right)}{\lambda}+1\right) \pi}{\sqrt{f^{\prime}\left(r_{H}\right) g^{\prime}\left(r_{H}\right)}} \lambda E\right], \quad \Gamma_{\text {in }} \propto \exp \left[-2 \frac{\left(\frac{C\left(r_{H}\right)}{\lambda}-1\right) \pi}{\sqrt{f^{\prime}\left(r_{H}\right) g^{\prime}\left(r_{H}\right)}} \lambda E\right] .
$$

It is interesting to note that $\Gamma_{\text {out }}, \Gamma_{\text {in }}$ are different from that in the Schwarzschild-like coordinate, but the total probability is

$$
\Gamma=\frac{\Gamma_{\text {out }}}{\Gamma_{\text {in }}} \propto \exp \left[-\frac{4 \pi}{\sqrt{f^{\prime}\left(r_{H}\right) g^{\prime}\left(r_{H}\right)}} \lambda E\right]
$$

and the Hawking temperature (20) is also recovered.

ii) If $C\left(r_{H}\right)=\lambda$, we can write $C(r(u))=\lambda+H(r(u)) \sqrt{f(r(u)) g(r(u))}$, where $H(r(u))$ is a regular function at horizon. Then we have $G(r(u))=\frac{\lambda}{\sqrt{f(r(u)) g(r(u))}}+H(r(u))+D(r(u))$. Substituting it into $g_{u u}$ of metric (11), we find that there is no coordinate singularity at horizon $r_{H}$ now. From Eq. (26), we obtain

$$
\operatorname{Im} W_{+}(u)=\frac{2 \pi}{\sqrt{f^{\prime}\left(r_{H}\right) g^{\prime}\left(r_{H}\right)}} \lambda E, \quad \operatorname{Im} W_{-}(u)=0
$$


this implies that $\Gamma_{i n}=1$. So the overall tunnelling probability is

$$
\Gamma=\Gamma_{\text {out }} \propto \exp \left[-2 \operatorname{Im} W_{+}\right]=\exp \left[-\frac{4 \pi E}{\sqrt{f^{\prime}\left(r_{H}\right) g^{\prime}\left(r_{H}\right)}}\right] .
$$

It is obviously that the Hawking temperature (20) is recovered.

From above discussions we know that the Hawking temperature of general static spherically symmetric black hole arising from the scalar particles tunnelling is invariant in the general coordinate (11).

\section{TEMPERATURE OF GENERAL STATIC SPHERICALLY SYMMETRIC BLACK HOLE FROM DIRAC PARTICLES TUNNELLING}

In this section, we study the Dirac particles tunnelling of the black hole in the coordinates (9) and (11).

\section{A. Dirac particles tunnelling in Schwarzschild-like coordinate}

For a general background spacetime, the Dirac equation is [25]

$$
\left[\gamma^{\alpha} e_{\alpha}^{\mu}\left(\partial_{\mu}+\Gamma_{\mu}\right)+\frac{m}{\hbar}\right] \psi=0
$$

with

$$
\Gamma_{\mu}=\frac{1}{8}\left[\gamma^{a}, \gamma^{b}\right] e_{a}^{\nu} e_{b \nu ; \mu}
$$

where $\gamma^{a}$ are the Dirac matrices and $e_{a}^{\mu}$ is the inverse tetrad defined by $\left\{e_{a}^{\mu} \gamma^{a}, \quad e_{b}^{\nu} \gamma^{b}\right\}=2 g^{\mu \nu} \times 1$. For the general static spherically symmetric black hole in the Schwarzschild-like metric (9) the tetrad can be taken as

$$
e_{a}^{\mu}=\operatorname{diag}\left(\frac{1}{\sqrt{f(r)}}, \sqrt{g(r)}, \frac{1}{\sqrt{R(r)}}, \frac{1}{\sqrt{R(r)} \sin \theta}\right) .
$$

We employ the following ansatz for the Dirac field

$$
\begin{aligned}
& \psi_{\uparrow}=\left(\begin{array}{c}
A\left(t_{s}, r, \theta, \varphi\right) \xi_{\uparrow} \\
B\left(t_{s}, r, \theta, \varphi\right) \xi_{\uparrow}
\end{array}\right) \exp \left(\frac{i}{\hbar} I_{\uparrow}\left(t_{s}, r, \theta, \varphi\right)\right)=\left(\begin{array}{c}
A\left(t_{s}, r, \theta, \varphi\right) \\
0 \\
B\left(t_{s}, r, \theta, \varphi\right) \\
0
\end{array}\right) \exp \left(\frac{i}{\hbar} I_{\uparrow}\left(t_{s}, r, \theta, \varphi\right)\right), \\
& \psi_{\downarrow}=\left(\begin{array}{c}
C\left(t_{s}, r, \theta, \varphi\right) \xi_{\downarrow} \\
D\left(t_{s}, r, \theta, \varphi\right) \xi_{\downarrow}
\end{array}\right) \exp \left(\frac{i}{\hbar} I_{\downarrow}\left(t_{s}, r, \theta, \varphi\right)\right)=\left(\begin{array}{c}
0 \\
C\left(t_{s}, r, \theta, \varphi\right) \\
0 \\
D\left(t_{s}, r, \theta, \varphi\right)
\end{array}\right) \exp \left(\frac{i}{\hbar} I_{\downarrow}\left(t_{s}, r, \theta, \varphi\right)\right),
\end{aligned}
$$

where "†" and " $\downarrow$ " represent the spin up and spin down cases, and $\xi_{\uparrow}$ and $\xi_{\downarrow}$ are the eigenvectors of $\sigma^{3}$. Inserting Eqs. (32), (33) into the Dirac equation (31) and employing

$$
I_{\uparrow}=-E t_{s}+W(r)+J(\theta, \varphi),
$$


to the lowest order in $\hbar$ we obtain

$$
\begin{aligned}
& -\frac{A}{\sqrt{f(r)}} E+\sqrt{g(r)} B W^{\prime}(r)+m A=0, \\
& \frac{B}{\sqrt{R(r)}}\left(J_{\theta}+\frac{i}{\sin \theta} J_{\varphi}\right)=0, \\
& \frac{B}{\sqrt{f(r)}} E-\sqrt{g(r)} A W^{\prime}(r)+m B=0, \\
& -\frac{A}{\sqrt{R(r)}}\left(J_{\theta}+\frac{i}{\sin \theta} J_{\varphi}\right)=0,
\end{aligned}
$$

where we consider only the positive frequency contributions without loss of generality. Eqs. (36) and (38) both yield $\left(J_{\theta}+\frac{i}{\sin \theta} J_{\varphi}\right)=0$ regardless of $A$ or $B$, implying that $J(\theta, \varphi)$ must be a complex function. We therefore can ignore $J$ from this point (or else pick the trivial $J=0$ solution).

Consider first the massless case $m=0$, Eqs. (35) and (37) give

$$
W_{ \pm}(r)= \pm \int \frac{E d r}{\sqrt{f(r) g(r)}}
$$

We therefore recover the expected Hawking temperature (20) in the massless case.

In the massive case $m \neq 0$, Eqs. (35) and (37) show

$$
\left(\frac{A}{B}\right)^{2}=\frac{\frac{E}{\sqrt{f(r)}}+m}{\frac{E}{\sqrt{f(r)}}-m}
$$

and

$$
W_{ \pm}(r)=\int \frac{E}{\sqrt{f(r) g(r)}} \frac{2\left(\frac{A}{B}\right)}{1+\left(\frac{A}{B}\right)^{2}} d r
$$

Noting $\lim _{r \rightarrow r_{H}}\left(\frac{A}{B}\right)^{2}=1$, we find that the result of integrating around the pole for $W$ in the massive case is the same as the massless case and we recover the Hawking temperature (20).

For the spin-down case the calculation is very similar to the spin-up case discussed above. Other than some changes of sign, the equations are of the same form as the spin up case. For both the massive and massless spin down cases the Hawking temperature (20) is obtained, implying that both spin up and spin down particles are emitted at the same temperature.

\section{B. Dirac particles tunnelling in general coordinate}

We take

$$
\begin{aligned}
& \psi_{\uparrow}=\left(\begin{array}{c}
A(v, u, \theta, \varphi) \xi_{\uparrow} \\
B(v, u, \theta, \varphi) \xi_{\uparrow}
\end{array}\right) \exp \left(\frac{i}{\hbar} I_{\uparrow}(v, u, \theta, \varphi)\right)=\left(\begin{array}{c}
A(v, u, \theta, \varphi) \\
0 \\
B(v, u, \theta, \varphi) \\
0
\end{array}\right) \exp \left(\frac{i}{\hbar} I_{\uparrow}(v, u, \theta, \varphi)\right), \\
& \psi_{\downarrow}=\left(\begin{array}{c}
C(v, u, \theta, \varphi) \xi_{\downarrow} \\
D(v, r, \theta, \varphi) \xi_{\downarrow}
\end{array}\right) \exp \left(\frac{i}{\hbar} I_{\downarrow}(v, u, \theta, \varphi)\right)=\left(\begin{array}{c}
0 \\
C(v, u, \theta, \varphi) \\
0 \\
D(v, u, \theta, \varphi)
\end{array}\right) \exp \left(\frac{i}{\hbar} I_{\downarrow}(v, u, \theta, \varphi)\right)
\end{aligned}
$$


where $I_{\uparrow}=-E v+W(u)+J(\theta, \varphi)$. For the line element (11), we chose the tetrad

$$
e_{a}^{\mu}=\left(\begin{array}{cccc}
\frac{\lambda}{\sqrt{f(r(u))}} & \sqrt{g(r(u))} G(r(u)) & 0 & 0 \\
0 & \sqrt{g(r(u))} F(r(u)) & 0 & 0 \\
0 & 0 & \frac{1}{\sqrt{R(r(u))}} & 0 \\
0 & 0 & 0 & \frac{1}{\sqrt{R(r(u))} \sin \theta}
\end{array}\right) .
$$

Then the Dirac equation (31) can be expressed as

$$
\begin{gathered}
{\left[-\frac{\lambda}{\sqrt{f(r(u))}} A-\sqrt{g(r(u))} G(r(u)) B\right] E+\sqrt{g(r(u))} F(r(u)) B W^{\prime}(u)+m A=0,} \\
{\left[\frac{\lambda}{\sqrt{f(r(u))}} B+\sqrt{g(r(u))} G(r(u)) A\right] E-\sqrt{g(r(u))} F(r(u)) A B W^{\prime}(u)+m B=0 .}
\end{gathered}
$$

For the case $m=0$, we find

$$
W^{\prime}(u)=\left[\frac{G(r(u))}{F(r(u))} E \pm \frac{\lambda E}{\sqrt{f(r(u)) g(r(u))} F(r(u))}\right],
$$

which is similar to Eq. (23). Taking the same method used in the section III, it is easy to get the Hawking temperature (20).

For the case $m \neq 0$, we find

$$
\left(\frac{A}{B}\right)^{2}=\frac{\frac{E}{\sqrt{f(r(u))}}+m}{\frac{E}{\sqrt{f(r(u))}}-m}
$$

and $\lim _{u \rightarrow u_{H}} \frac{A}{B}= \pm 1$. We have

$$
W^{\prime}(u)=\left[\frac{G(r(u))}{F(r(u))} E \pm \frac{2 \lambda\left|\frac{A}{B}\right| E}{\sqrt{f(r(u)) g(r(u))} F(r(u))\left(\frac{A^{2}}{B^{2}}+1\right)}\right],
$$

which is similar to Eq. (23). Taking the same method used in the section IIB, we also find the same Hawking temperature (20).

From above discussions we know that the Hawking temperature of general static spherically symmetric black hole arising from the Dirac particles tunnelling is also invariant in the general coordinate (11).

\section{SUMMARY}

The Hawking temperature of the Schwarzschild black hole in the isotropic coordinate shows us that the temperature is not invariant. What kinds of coordinate can keep the Hawking temperature invariant for the general static spherically symmetric black hole? By studying the Hawking radiation of the most general static spherically symmetric black hole arising from scalar and Dirac particles tunnelling, we find that it is invariant in the general coordinate representation (11), which satisfies two conditions: a) its radial coordinate transformation is regular at the event horizon; and b) there is a time-like Killing vector.

We also find some other interesting results: 1) For the coordinate representations which do not exist coordinate singularity, such as the general coordinate (11) with $C\left(r_{H}\right)=\lambda$ (include the Painlevé (12) and Lemaitre (13)), 
$W_{+}$has a pole at the event horizon but $W_{-}$has a well defined limit at the horizon. Then the imaginary part of $W_{-}$is zero due to the imaginary parts of the action can only come from the pole and the probability of a particle tunnelling from inside to outside the horizon is described by $\Gamma=\Gamma_{\text {out }}$. 2) The mass of the particles and the angular quantum number do not affect the Hawking temperature for both scalar and Dirac particles. 3) When time coordinate transforms from $t_{s}$ to $\lambda t_{s}$, i. e., we re-scale the time, the corresponding energy $E$ of the total tunnelling particles is increased by $\lambda$ times, so re-scale of the time does not affect the Hawking temperature.

\section{Acknowledgments}

This work was supported by the National Natural Science Foundation of China under Grant No. 10675045; the FANEDD under Grant No. 200317; the Hunan Provincial Natural Science Foundation of China under Grant No. 08JJ0001; and the construct program of the key discipline in hunan province.

[1] R. Kerner and R. B. Mann, Phys. Rev. D73 104010 (2006).

[2] R. Kerner and R. B. Mann, arXir: 0710. 0612.

[3] M. K. Parikh and F. Wilczek, Phys. Rev. Lett. 855042 (2000).

[4] M. Agheben, M. Nadalini, VanzoL, and S. Zerbini, J. High Energy Phys. 0505 (2005) 014.

[5] S. Shankaranarayanan and K. Srinivasan and T. Padmanabhan, Mod. Phys. Lett. A 16 571 (2001).

[6] S. Shankaranarayanan and T. Padmanabhan and K. Srinivasan, Class. Quantum Grav. 192671 (2002).

[7] K. Srinivasan and T. Padmanabhan, Phys. Rev. D 60024007 (1999).

[8] M. Nadalini, L. Vanzo and S. Zerbini, J. Phys. A: Math. Gen. 396601 (2006).

[9] E. C. Vagenas, Nuovo Cim. 117B, 899 (2002).

[10] M. K. Parikh, Phys. Lett. B 546189 (2002).

[11] M. K. Parikh, Int. J. Mod. Phys. D13 2351 (2004).

[12] M. K. Parikh, arXiv: hep-th/0402166.

[13] P. Kraus, and Frank Wilczek, gr-qc/9406042

[14] P. Kraus and F. Wilczek, Nucl. Phys. B433 403 (1995).

[15] P. Kraus and F. Wilczek, Nucl. Phys. B437 231 (1995).

[16] M. Arzano, A. Medved and E. Vagenas, J. High Energy Phys. 0509 (2005) 037.

[17] Qing-Quan Jiang, Shuang-Qing Wu, and Xu Cai, Phys.Rev. D73 064003 (2006).

[18] Jingyi Zhang, and Zheng Zhao, Phys. Lett. B638 110 (2006).

[19] Shuang-Qing Wu, and Qing-Quan Jiang, J. High Energy Phys. 0603 (2006) 079.

[20] A. J. M. Medved and E. Vagenas, Mod. Phys. Lett. A20 2449 (2005).

[21] Bhramar Chatterjee, Amit Ghosh, P. Mitra, arXiv: 0704. 1746.

[22] P. Mitra, Phys. Lett. B648 240 (2007).

[23] C. K. Ding and J. L. Jing, J. High Energy Phys. 09 (2007) 067.

[24] C. K. Ding and J. L. Jing, Chin. Phys Lett. 24 (2007) 2189 (2007).

[25] D. R. Brill and J. A. Wheeler, Rev. Mod. Phys. 29 (1995) 465 (1995). 